Article

\title{
Electromagnetic Transient-Transient Stability Analysis Hybrid Real-Time Simulation Method of Variable Area of Interest
}

\author{
Bingda Zhang *, Shipei Nie and Zhao Jin \\ The Key Laboratory of Smart Grid of Ministry of Education, Tianjin University, Tianjin 300072, China; \\ spn2018@tju.edu.cn (S.N.); 1017234013@tju.edu.cn (Z.J.) \\ * Correspondence: bdzhang@tju.edu.cn; Tel.: +86-022-2740-4101
}

Received: 14 September 2018; Accepted: 29 September 2018; Published: 1 October 2018

\begin{abstract}
To make the object of electromagnetic transient (EMT) simulation flexible to change, the authors propose using the method of electromagnetic transient-transient stability analysis (TSA) hybrid real-time simulation of the variable area of interest. The area where the fault is to be set, or where the operation takes place, is defined as the area of interest. The simulation object is divided into multiple sub-networks. The EMT simulation range is determined according to the voltage drop depth at the boundary of the adjacent sub-network caused by the three-phase short-circuit fault at the boundary of an area of interest. The Norton equivalent is obtained by using the sub-network as a basic unit. The electromagnetic sub-network forms its own Norton equivalent on the TSA side by means of the Norton equivalent admittance of its TSA model. Based on this, the overall framework of hybrid real-time simulation of the variable area of interest is constructed. The fundamental phasor prediction and Norton equivalent current source prediction are adopted to reduce the interface error. The performance of the proposed method in terms of feasibility, flexibility, and effectiveness have been verified by the simulation studies on the IEEE 118-bus system.
\end{abstract}

Keywords: variable area of interest; hybrid real-time simulation; network partitioning scheme; generality; Norton equivalent; prediction

\section{Introduction}

Real-time (RT) simulation means that the time that is required by the simulation platform to complete the computation of state outputs for each step size is shorter than or equal to the selected step size [1], which can be used for design, prototype construction, hardware-in-the-loop testing, teaching, and training [2]. Electromagnetic transient (EMT) simulation generally calculates with a 30-100 $\mu$ s time-step [3], which is mainly used for closed-loop testing of control systems, such as static var compensators (SVCs), synchronous condensers, and protection devices [4]. Although it is considered to be more precise, its simulation calculation burden is heavy, which limits the simulation scale [5]. The typical calculation time-step of transient stability analysis (TSA) simulation is 8-10 ms [2]. Although it sacrifices the description of fast transients, harmonics, and electromagnetic nonlinearities, it can be used for closed-loop testing of power flow controllers and energy storage equipment, and dispatcher training, etc. TSA has the advantage of large system analysis [2,4-6], therefore, it is not always necessary to perform EMT simulation on the whole system, only the part that needs detailed study. When the computational resources of the large-scale power system for EMT simulation are insufficient, a feasible solution is that the part requiring detailed study performs EMT simulation and the rest perform TSA simulation. Both are connected through an interface.

Interface technology is the key to hybrid simulation [7]. The interface technology of hybrid simulation mainly involves three aspects: equivalent method, interaction timing, and interface position 
selection. Regarding the equivalent method, when simulating the TSA sub-system, the EMT sub-system is generally replaced by a power source [7-9], a current source [10-12], or a Thevenin (Norton) equivalent [13]. The power source equivalent is the most common EMT sub-system equivalent in the current EMT-TSA hybrid simulation. The current source equivalent is the most direct among the three equivalent methods. Its basic idea is to measure the phasor of the fundamental wave current of each sequence and directly transmit it to the TSA side [14]. When simulating the EMT sub-system, the TSA sub-system is generally replaced by the Thevenin (Norton) equivalent, which is based on fundamental frequency [14]. Regarding interaction timing, in view of the shortcomings that the interface error of current parallel data interaction mode is large, an improved parallel data interaction scheme is presented [15]. The core of the data interaction scheme is to use the EMT sub-system historical calculation results to predict the parameters that are required in the current iterative calculation of the TSA subprogram. To reduce the influence of communication delay on simulation accuracy, an interface electrical quantity interaction method, which is based on the principle of using the other party's simulation data as early as possible, is proposed [16]. When considering interface location selection, there are currently two ideas for developing network partition schemes: To reduce the modeling amount of the electromagnetic sub-system, the interface position is often set at the DC converter bus $[17,18]$. According to the need to partition the network at a low asymmetry position, the interface position is extended to the TSA side [10,19]. However, existing network partition schemes cannot be used for pure AC power networks. Concerning accuracy, hybrid simulation is more suitable for an AC system [20-22], so the network partition scheme for an AC system is very important. Some work has pointed out that, for the actual large-scale interconnected power grid, the network partitioning scheme should analyze the coupling relationship between the various parts of the system analytically and carry out the network partition scheme in the position where the system coupling relationship is most clear [23], however, no specific practice is suggested.

Following the target power system being carried out to network partitioning in the existing works, the areas of EMT simulation and TSA simulation are often fixed. To make the area of EMT simulation flexible to change, the current authors propose the method of hybrid real-time simulation of the variable area of interest in this paper. That is, according to the difference of interest areas, EMT simulation of different areas is performed in different periods, and TSA simulation is performed on other areas. The authors divide the simulation object into multiple sub-networks. The EMT simulation range of an area of interest is determined according to the voltage drop depth at the boundary of the adjacent sub-network that is caused by the three-phase short-circuit fault at the boundary of each area of interest. According to the characteristics of a hybrid real-time simulation of variable area of interest, the Norton equivalent is obtained by using the sub-network as a basic unit. The calculations on the electromagnetic side and the TSA side mainly include three steps. Based on this, a general model of the contact system is constructed. The contact system consists of the Norton equivalents of electromagnetic sub-networks and the Norton equivalents of the TSA models of the remaining sub-networks. The electromagnetic sub-network forms its own Norton equivalent model on the TSA side by means of the positive sequence components of voltage and current that are extracted by the discrete sequence at its interfaces and the Norton equivalent admittance of its TSA model. Looking at the problem of large interface error in the parallel data interaction mode, which is caused by the use of the previous time-step equivalent parameters on both the TSA side and the electromagnetic side [15], the fundamental phasor prediction and the Norton equivalent current source prediction are used to reduce the interface error.

The rest of the paper is organized as follows. The simulation object is divided into multiple sub-networks and a network partition scheme is provided in Section 2. Section 3 presents the overall framework of hybrid real-time simulation of the variable area of interest. Section 4 details the relevant practices of the TSA side. Section 5 presents the specific practices of the EMT side. Section 6 presents the case studies and the simulation results. Finally, brief conclusions are made in Section 7. 


\section{Network Partition Scheme}

To facilitate the switching between an electromagnetic transient (EMT) program and the corresponding transient stability analysis (TSA) program and to determine the EMT simulation range, the authors first divide the large power network into multiple sub-networks. Based on this, the EMT simulation range corresponding to each sub-network is determined. The simulation range does not change with different faults or operations in the sub-network.

\subsection{Determination of the Sub-Networks}

To avoid excessive writing of EMT programs and to avoid the situation that obtaining an equivalent model and calculating the contact system, which will cause an excessive additional amount of calculation (relative to the case of no network segmentation) in the case of an excessive number of interfaces, the sub-network that is generated by division cannot be too small. Since the target system is large, the interface position is only set at the boundary. When a fault or operation occurs at the boundary, the surrounding sub-networks are included in the scope of EMT simulation. When the sub-network that is generated by division is too large, it is not conducive to the improvement of simulation efficiency. Therefore, the sub-network that is generated by division should not be too small or too large. Under the above principle, the simulation object is divided into multiple sub-networks, each of which is an area of interest.

\subsection{Determination of EMT Simulation Range}

The lighter the voltage drops, the lower the harmonic content, and TSA simulation only considers the fundamental frequency component. Therefore, when the fault occurs, the voltage drop depth can be used to determine the affiliated area corresponding to each sub-network. The affiliated area is an area that extends outward to ensure the accuracy of the simulation of the area of interest. The sub-network and its affiliated area are the EMT simulation range corresponding to each sub-network. The voltage drop depth is defined as:

$$
\delta_{U}=\frac{U_{0}-U_{1}}{U_{0}}
$$

where $U_{0}$ and $U_{1}$ are the voltage amplitudes before and after the fault, respectively.

Since the three-phase short-circuit fault has the widest influence range under the same conditions, a three-phase short-circuit fault can be set at the boundary of each sub-network, and the voltage drop depth $\delta_{U b}$ at the fault location is used as a reference. When the ratio of $\delta_{U}$ to $\delta_{U b}$ of nodes at adjacent boundaries is less than $\delta_{t h}$ (threshold), then the impact of faults on these nodes is negligible. These nodes at the boundary can be used as interface nodes. When the ratio is larger than $\delta_{t h}$, the remaining boundary nodes that are closer to the fault location should be detected again until all of the nodes that meet the requirements are found. This will determine the range of EMT simulation.

\section{Overall Framework of Hybrid Real-Time Simulation of Variable Area of Interest}

Real-time simulation needs to meet the requirements of hardware closed loop and real-time, therefore, the overall protocol for system interaction is shown in Figure 1. Regarding hardware-in-the-loop, the relevant output information of the electromagnetic side is written into the shared data area in a small time-step, the relevant output information on the TSA side is written into the shared data area in a large time-step and the output information that is processed by the input/output program is transmitted to the hardware equipment. The input/output program is a program that processes input and output data. The information input by the hardware device is written to the shared data area after being processed by the input/output program. The EMT and TSA calculation processes can read the required information from the shared data area. TSA simulation is performed with a large time-step size $H$ and EMT with a small time-step size $h$. The two are performed in parallel. The shared data area contains multiple storage units. 


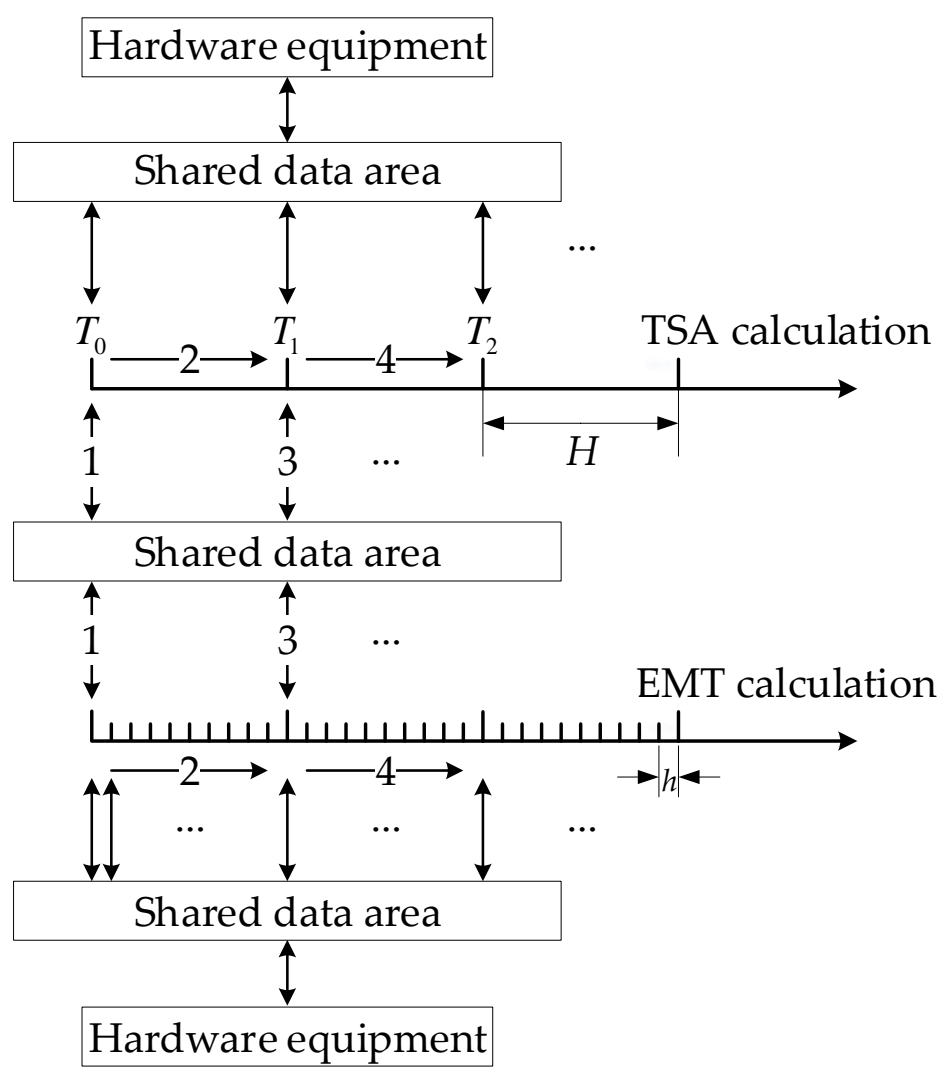

Figure 1. The overall protocol for a hybrid real-time simulation system interaction. TSA: transient stability analysis; EMT: electromagnetic transient.

To make the areas of EMT and TSA simulation flexible to change, the external equivalent model is obtained from the sub-network.

While considering the characteristics of a hybrid real-time simulation of a variable area of interest, the model of the contact system needs to be kept unchanged, therefore, it is necessary to construct a general model for the calculation of the contact system. To this purpose, the calculations on the electromagnetic and TSA sides mainly include three steps: obtaining the equivalence models needed to build the contact system, the calculation of the contact system, and the calculation of each sub-network itself.

TSA simulation is performed on all sub-networks, and parallel calculation is performed between sub-networks. The sub-networks within the scope of the EMT sub-system perform both EMT and TSA simulation.

The information interaction diagram of the whole system is shown in Figure 2. $S_{k}(k=$ $1, \ldots, i, \ldots, i+m, \ldots, n)$ represents the $k$-th sub-network in Figure 2 . The area where $S_{k}$ is located is the model area. The TSA model of all sub-networks completes obtaining the equivalent parameters of the TSA sub-networks, the attaining of the equivalent parameters II of electromagnetic sub-networks, and the calculation of the TSA sub-networks themselves. The EMT model of the sub-networks completes the acquiring of the equivalent parameters I of electromagnetic sub-networks and the calculation of the electromagnetic sub-networks themselves. Both $D_{K E}$ and $D_{k \mathrm{E}}^{\prime}$ represent the equivalent parameters of the $k$-th sub-network, but they represent different meanings. $I_{k S}$ represents the voltage and current values at the $k$-th sub-network interface (the interface parameters). The interface parameters are calculated by the contact system. The interface parameters on the TSA side are used for the calculation of the TSA sub-networks themselves. The electromagnetic side interface parameters are used not only for the calculation of the electromagnetic sub-networks themselves, but also for the phasor extraction of the TSA side. The area where $D_{K E}, D_{k E^{\prime}}^{\prime}$ and $I_{k S}$ are located is the data area. To use 
the parameters conveniently, the relevant parameters on both sides are stored in different storage units by sub-network number. The components in the ellipse belong to the electromagnetic side. The sub-network within the solid ellipse is the area of interest, and the sub-network within the dashed ellipse is the affiliated area. The area of interest contains only one sub-network, while the affiliated area might contain multiple sub-networks. The components in the rectangle belong to the TSA side. Arrows indicate the process of information interaction. To describe more easily, the "equivalent parameters of TSA sub-networks" appears twice in Figure 2, but their parameters and storage addresses are identical. The contact system exists in the form of phasors in the process of TSA calculation. It is recorded as the TSA model of the contact system. The contact system exists in the form of instantaneous value in the EMT calculation process. It is recorded as the EMT model of the contact system. The role of the pre-processing of the TSA sub-network equivalent parameters is to predict the equivalent current sources (or equivalent voltage sources) of the next time-step of the TSA sub-networks and to realize the discretization of the equivalent current sources (or equivalent voltage sources) according to the electromagnetic step size.

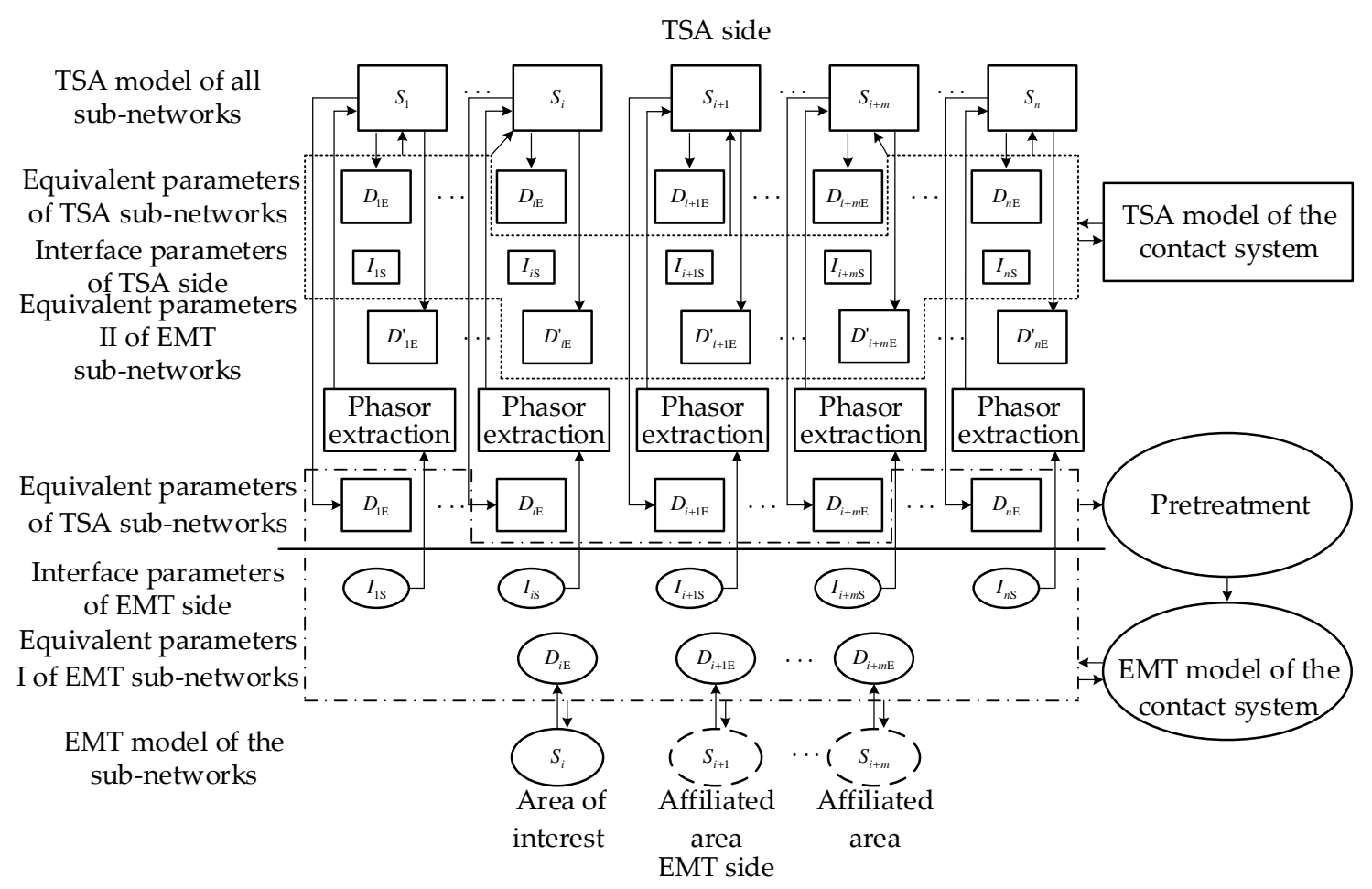

Figure 2. Information exchange diagram of a hybrid real-time simulation of a variable area of interest.

Only one of the equivalent parameters that were obtained from the same sub-network under different modeling methods is used by the corresponding contact system. The sub-networks $S_{i} \ldots$ $S_{i+m}$ that perform EMT simulation are variable. The EMT model of the sub-networks vary with the difference of the areas that perform EMT simulation. The TSA model of all sub-networks, the TSA model of the contact system, and the EMT model of the contact system are invariant. Their storage space is also invariant and only the parameters of them are changed. The interface parameters of the EMT side and the equivalent parameters of the TSA sub-networks are stored in a plurality of storage units for ping-pong operations between the EMT side and TSA side, respectively. The ping-pong frequencies on both sides are $H$, and the data of two or more time-steps of the same parameter can be saved. To write programs conveniently, the voltages and the currents at all sub-network interfaces on the electromagnetic side are carried out via phasor extraction. 


\section{The Simulation of TSA Side}

To ensure the consistency of the external characteristics of the TSA and EMT models of the same component in steady state, and to obtain the equivalent model of the TSA sub-network easily, the component models should be simplified and preprocessed. The load adopts the constant impedance model. The line adopts the pi-type, quasi-steady-state model. The transformer is the ideal transformer and it adopts the pi-type equivalent model [24]. The generator uses a six-order detailed model and it is processed as a virtual injection current in parallel with a virtual admittance.

\subsection{The Construction Method of TSA General Model of Contact System}

The TSA model and the EMT model of the same sub-network have the same external equivalent form, so the models of the contact system are unchanged. Second, when the EMT and TSA sub-systems are equivalent to the first-order boundary condition (the Thevenin or Norton equivalent), the iterative factor is the smallest under the same conditions, which is beneficial to accelerate the convergence of the system iteration [25], since fast convergence is critical for real-time simulation applications. Therefore, both the TSA sub-network and the EMT sub-network are replaced by a Norton equivalent.

The Norton equivalents of the TSA model of all sub-networks are obtained according to the method [13]. The Norton equivalent of an EMT sub-network is derived from the modified Norton equivalent of the TSA model of the sub-network. Since the corresponding TSA sub-network is applied with the same fault or operation at the same time when the EMT sub-network fails or operates, the Norton admittance $Y_{t s a}^{\prime}$ of the corresponding TSA model does not require correction. Only the Norton equivalent current source of its TSA model needs to be corrected. The correction method is as follows.

According to the discrete sequence that was calculated by the EMT model of the contact system, the voltage positive sequence component $\vec{U}_{1}$ at the interface and the current positive sequence component $\vec{I}_{1}$ of each branch are obtained by fitting. According to $\vec{U}_{1}, \vec{I}_{1}$, and the equivalent admittance $Y_{t s a}$ at the interface, the modified Norton current source $\vec{I}_{m}$ is given by:

$$
\vec{I}_{m}=\vec{I}_{1}-Y_{t s a}^{\prime} \vec{U}_{1}
$$

\subsection{Phasor Extraction}

To reduce the computational burden on the electromagnetic side, the phasor extraction is performed on the TSA side. The phasor is extracted by the method $[26,27]$ in this paper. The phasors are extracted from the voltage and current waveforms at the boundary buses, which are provided by EMT simulation. To be clear, the presentation deals with currents, but the processing of the voltages is similar.

The amplitude and the phase angle of the current positive sequence component are computed from the three time-varying current waveforms by projecting the latter on $(x, y)$ reference axes. These are the axes used in the TSA simulation to project the rotating vectors that are associated with quasi-sinusoidal variables and obtain their corresponding rectangular components. This is shown in Figure 3 , where $I_{x}$ and $I_{y}$ are the components of phasor $\vec{I}_{1}$, all three varying with time. 


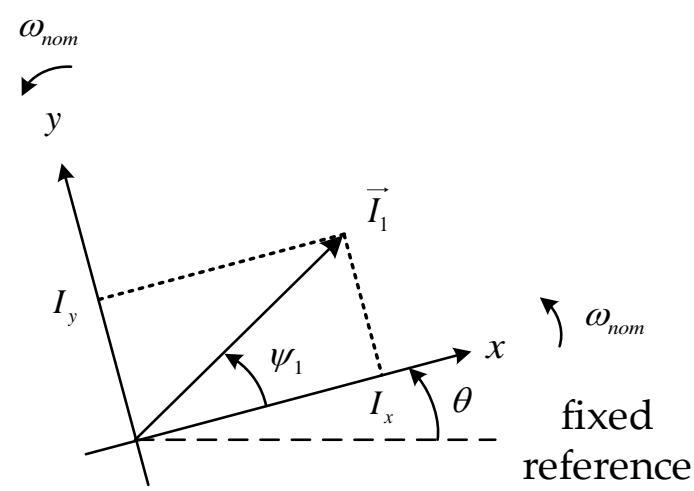

Figure 3. Reference axes involved in the phasor extraction.

The reference axes are taken as rotating at the angular velocity $\omega_{\text {nom }}$ in the TSA simulation. Thus, at time $t$, the angle between the $x$-axis and a fixed reference is (see Figure 3):

$$
\theta=\omega_{\text {nom }} t
$$

assuming that the $x$-axis and the reference axis coincide at $t=0$.

The vector of projected currents, $i_{x y}$, is obtained from the three-phase instantaneous currents, $i_{a b c}$, using the linear transformation:

$$
i_{x y}=T i_{a b c}
$$

where:

$$
T=\frac{\sqrt{2}}{3}\left[\begin{array}{ccc}
\cos \theta & \cos \left(\theta-\frac{2 \pi}{3}\right) & \cos \left(\theta-\frac{4 \pi}{3}\right) \\
-\sin \theta & -\sin \left(\theta-\frac{2 \pi}{3}\right) & -\sin \left(\theta-\frac{4 \pi}{3}\right)
\end{array}\right],
$$

When the three-phase currents were balanced at the fundamental frequency, for example:

$$
i_{a b c}=\left[\begin{array}{c}
i_{a} \\
i_{b} \\
i_{c}
\end{array}\right]=\left[\begin{array}{c}
\sqrt{2} I_{1} \cos \left(\omega_{\text {nom }} t+\psi_{1}\right) \\
\sqrt{2} I_{1} \cos \left(\omega_{\text {nom }} t+\psi_{1}-\frac{2 \pi}{3}\right) \\
\sqrt{2} I_{1} \cos \left(\omega_{\text {nom }} t+\psi_{1}-\frac{4 \pi}{3}\right)
\end{array}\right],
$$

then, it is easy to conclude that:

$$
i_{x y}=\left[\begin{array}{c}
I_{x} \\
I_{y}
\end{array}\right]=\left[\begin{array}{c}
I_{1} \cos \psi_{1} \\
I_{1} \sin \psi_{1}
\end{array}\right],
$$

which shows that the components of $i_{x y}$ are indeed the projections on $x$ and $y$ of a vector rotating at angular velocity $\omega_{\text {nom }}$, having amplitude $I_{1}$, and a phase angle $\psi_{1}$ relative to the $x$-axis, as shown in Figure 3. $I_{x}$ and $I_{y}$ are the real and imaginary parts of the current phasor required in the TSA simulation, respectively.

This phasor extraction technique does not introduce a delay that is associated with the processing of the waveforms at times prior to $t+H$ [27].

Equation (7), however, is applicable only to balanced three-phase currents at a fundamental frequency. When the effects of a fault that is located in the EMT sub-system are felt at the boundary between the EMT and TSA sub-systems, the boundary current waveforms are affected by "noise", consisting of aperiodic, negative and zero-sequence components, and harmonics. Therefore, filtering is necessary to eliminate their effects.

The aperiodic (regarding the negative-sequence) components present in $i_{a b c}$ will show their effects on $i_{x y}$ in the form of fundamental (regarding double-fundamental) frequency sinusoidal components. The Butterworth low pass filter [28] can be used for filtering. 


\subsection{Fundamental Phasors Prediction}

Since the fundamental-frequency voltage and current phasors are extracted according to the interface information of the EMT side of the last TSA step size, if the phasors are directly used in the calculation of the Norton equivalent current sources, it will cause a large interface error. Interface errors can be reduced by predicting the fundamental voltage and current phasors. The presentation deals with the fundamental current phasors, but the fundamental voltage phasors are treated similarly. The details are as follows.

The amplitude $\widetilde{I}_{1}(t)$ and the phase angle $\widetilde{\psi}_{1}(t)$ of fundamental current phasors that are required for the calculation of the current step size are predicted based on the fundamental current phasors extracted by the current and the last time-steps of the TSA side. Concerning the first-order (linear) prediction, their values are given by the following equation:

$$
\left\{\begin{array}{l}
\widetilde{I}_{1}(t)=2 I_{1}(t)-I_{1}(t-H) \\
\widetilde{\psi}_{1}(t)=2 \psi_{1}(t)-\psi_{1}(t-H)
\end{array},\right.
$$

\section{The Simulation of EMT Side}

\subsection{The Construction Method of EMT General Model of Contact System}

The Norton equivalent of the electromagnetic sub-network is obtained by Gaussian elimination and obtaining the Norton equivalent of the remaining TSA sub-network needs the help of the Norton equivalent in phasor form of the TSA sub-network. The Norton equivalent in the form of an instantaneous value of the TSA sub-network can be obtained by the transformation of the relevant Norton equivalent parameters in the phasor form. The transformation is as follows: Norton equivalent admittance is converted into a form containing equivalent conductance and equivalent historical current source. The RL series branch is taken as an example to illustrate the process. The transformation process of more components can be found [29]. Concurrently, the Norton equivalent current source phasors are converted into an instantaneous value form.

The differential equation of the RL series branch is as follows:

$$
u(t)=L \frac{d i(t)}{d t}+R i(t)
$$

Equation (9) is differentiated by an implicit trapezoidal integration method and the result shows that:

$$
i(t)-i(t-h)=\frac{h}{2 L}[u(t-h)-R i(t-h)]+\frac{h}{2 L}[u(t)-R i(t)],
$$

where $u$ is the voltage at the interface and $i$ is the current in the equivalent impedance branch. Equation (10) is organized as:

$$
i(t)=\frac{2 L-h R}{2 L+h R} i(t-h)+\frac{h}{2 L+h R} u(t-h)+\frac{h}{2 L+h R} u(t),
$$

Equation (11) is rewritten as:

$$
i(t)=h_{s}(t-h)+G u(t)
$$

where:

$$
\left\{\begin{array}{l}
h_{S}(t-h)=\frac{2 L-h R}{2 L+h R} i(t-h)+\frac{h}{2 L+h R} u(t-h) \\
G=\frac{h}{2 L+h R}
\end{array},\right.
$$

$h_{s}(t-h), G$, respectively, represent the equivalent historical current source and the equivalent conductance after the transient calculation circuit is differentiated. 


\subsection{Norton Equivalent Current Source Prediction}

The update of the Norton equivalent current source from the TSA sub-network in the EMT model of the contact system is performed by the Norton equivalent current source prediction. Simultaneously, the Norton equivalent current source can be predicted to reduce the interaction error that is caused by the Norton equivalent current source of the TSA sub-network remaining constant within the interactive step size [30].

First, using linear extrapolation, the amplitude $\widetilde{I}_{t s a}(t+H)$ and phase angle $\widetilde{\phi}(t+H)$ of the next time-step Norton equivalent current sources are predicted based on the Norton equivalent current sources calculated by the current step size and the last step size of the TSA side. The predicted value is given by:

$$
\left\{\begin{array}{l}
\widetilde{I}_{t s a}(t+H)=2 I_{t s a}(t)-I_{t s a}(t-H) \\
\widetilde{\phi}(t+H)=2 \phi(t)-\phi(t-H)
\end{array},\right.
$$

Then, the Norton equivalent current source in the next time-step is linearly interpolated, thereby realizing the discretization of the Norton equivalent current source, according to the electromagnetic step size. The linear interpolation of the amplitude and phase angle of each phasor $\vec{I}_{t s a}$ is considered separately, and the details are as follows.

$H$ is assumed to be a multiple of $h, H=\rho h$ where $\rho$ is an integer. Occurring at the discrete time instant $t+k h(k=0, \ldots, \rho)$, the Norton current amplitude is given by:

$$
I_{t s a}(t+k h)=I_{t s a}(t)+\frac{k}{\rho}\left(\widetilde{I}_{t s a}(t+H)-I_{t s a}(t)\right),
$$

Similarly, the phase angle of the interpolation is given by:

$$
\phi(t+k h)=\phi(t)+\frac{k}{\rho}(\widetilde{\phi}(t+H)-\phi(t))
$$

Taking phase A as an example, the discretized Norton current is obtained as $(k=0, \ldots, \rho)$ :

$$
i_{a}(t+k h)=\sqrt{2} I_{t s a}(t+k h) \cos \left[\omega_{\mathrm{nom}}(t+k h)+\phi(t+k h)\right],
$$

$i_{a}(t+k h)$ can be used for the calculation of the EMT sub-system.

\section{Case Studies and Simulation Results}

The IEEE 118-bus system is taken as an example, and the example system is shown in Figure 4. A field programmable gate array (FPGA) is used as the simulation platform for testing. An EMT simulation is performed with $50 \mu \mathrm{s}$, and TSA simulation with $10 \mathrm{~ms}$. The implicit trapezoidal method is used for differentiating.

\subsection{The Network Partition Result}

Twenty-seven areas of interest are set in the IEEE 118-bus system; thus, 27 sub-networks are generated. To simplify, only partial network partitioning results are given. The nodes that are included in each sub-network are shown in Table 1 . The same nodes in every two sub-networks are boundary nodes. 


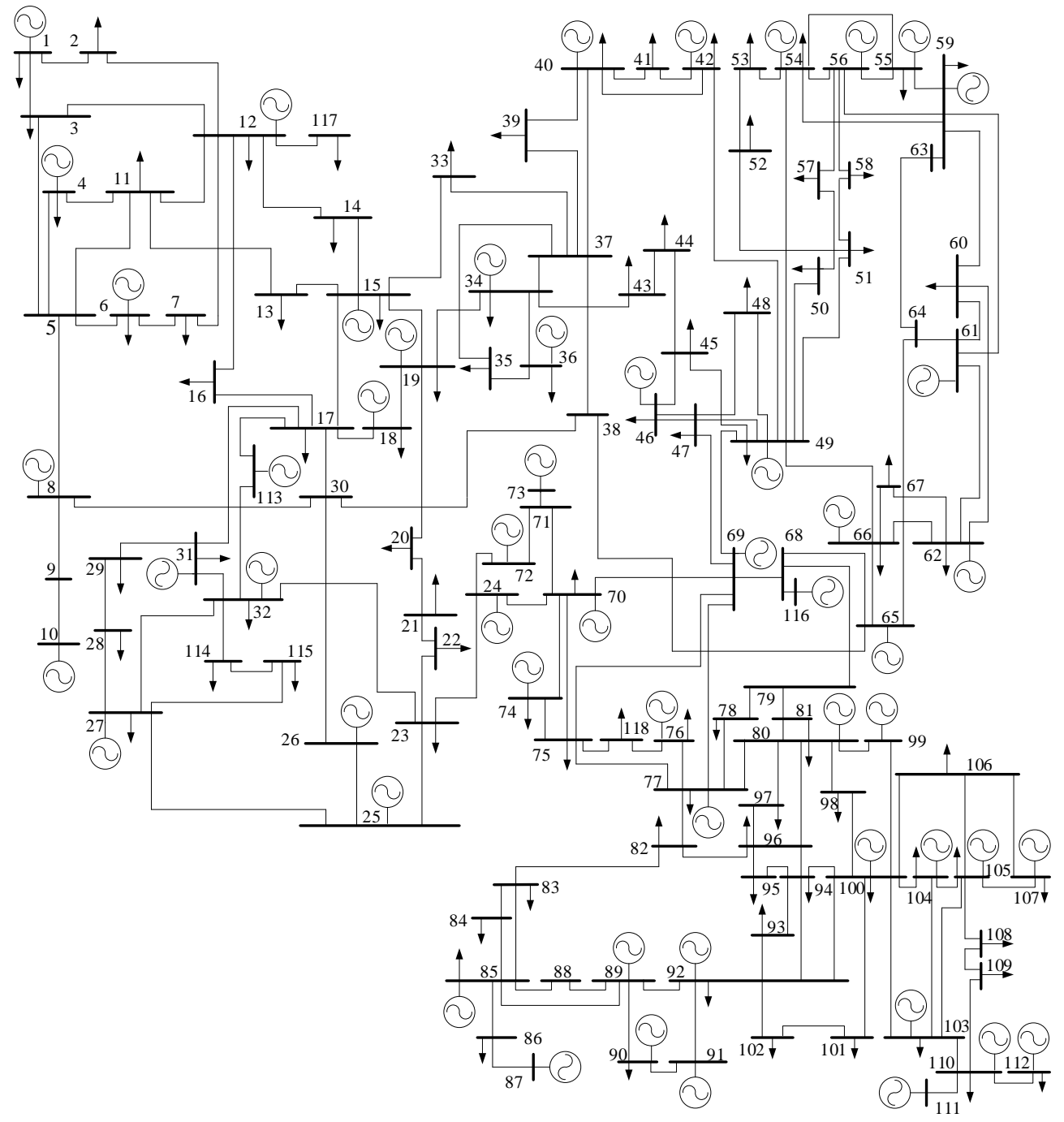

Figure 4. IEEE 118-bus system single line diagram.

Table 1. Partial sub-networks and their nodes.

\begin{tabular}{cc}
\hline Sub-Network $\boldsymbol{k}$ & Nodes Included in Sub-Network $\boldsymbol{k}$ \\
\hline 2 & $2,3,12,117,11,14,5,6,7,13,16$ \\
4 & $16,17,113,30,31,32,114,115,27,23$ \\
5 & $29,31,28,27$ \\
6 & $30,27,26,23,25$ \\
7 & $33,14,37,13,15,34$ \\
8 & $15,34,19,17,18,20,21,22,23$ \\
9 & $74,44,43,35,36,46,45,47,38,30$ \\
11 & $40,41,42,39,37,49$ \\
13 & $69,68,116,65,38,79$ \\
18 & $69,79,78,75,118,76,77,82,96$ \\
19 & $79,81,80,99,77,98,97,96,100$ \\
20 & $96,95,94,100,93,89,92,102,91$ \\
23 & $106,100,104,105,102,101,103$ \\
24 & $106,105,107,108$ \\
25 & $100,102,101,103,110,111$ \\
26 &
\end{tabular}


Subsequent to repeated experiments, to meet the simulation accuracy requirements, $\delta_{t h}$ can be taken to $0.03-0.034$, and, on this basis, the affiliated area corresponding to each sub-network can be determined. To simplify, only the affiliated areas of sub-networks 6, 7, and 20 are given. The affiliated area of sub-network 6 is sub-networks $4,5,8$, and 11 . The affiliated area of sub-network 7 is sub-networks $2,4,8,9$, and 13 . The affiliated area of sub-network 20 is sub-networks $18,19,23,24$, 26 , and 25.

\subsection{Verification and Application}

\subsubsection{Verification of Norton Equivalent Accuracy}

To illustrate the effectiveness of the Norton equivalent of the electromagnetic sub-network on the TSA side, three off-line simulation methods are designed for comparison: full EMT simulation, hybrid simulation with the electromagnetic sub-network using a Norton equivalent on the TSA side, and hybrid simulation with the electromagnetic sub-network using a current source equivalent on the TSA side. The full model PSCAD/EMTDC simulation method is represented by the symbol EMT, the hybrid simulation method using the Norton equivalent is represented by the symbol EMT + TSA + Norton, and the hybrid simulation method using the current source equivalent is represented by the symbol EMT + TSA + Current, in the following.

Sub-network 6 and its affiliated area (sub-networks 4, 5, 8, and 11) performs EMT simulation, and other parts perform TSA simulation. A $100 \mathrm{~ms}$ three-phase fault is applied to bus 23 . The phase A voltage of bus 23 and the rotor speed of the generator at bus 25 are as shown in Figure 5.

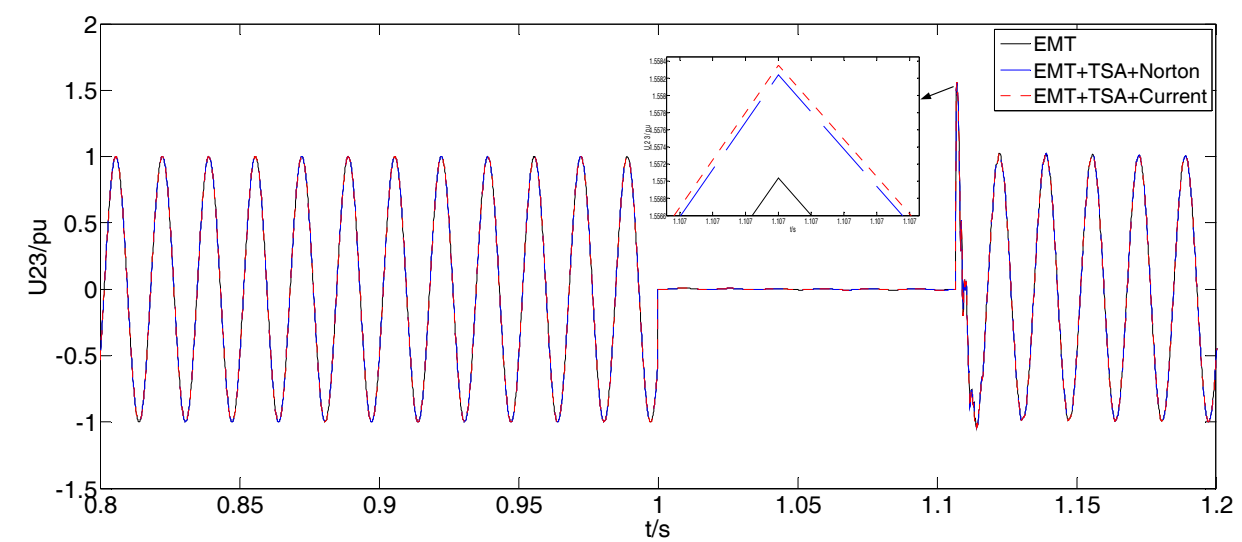

(a)

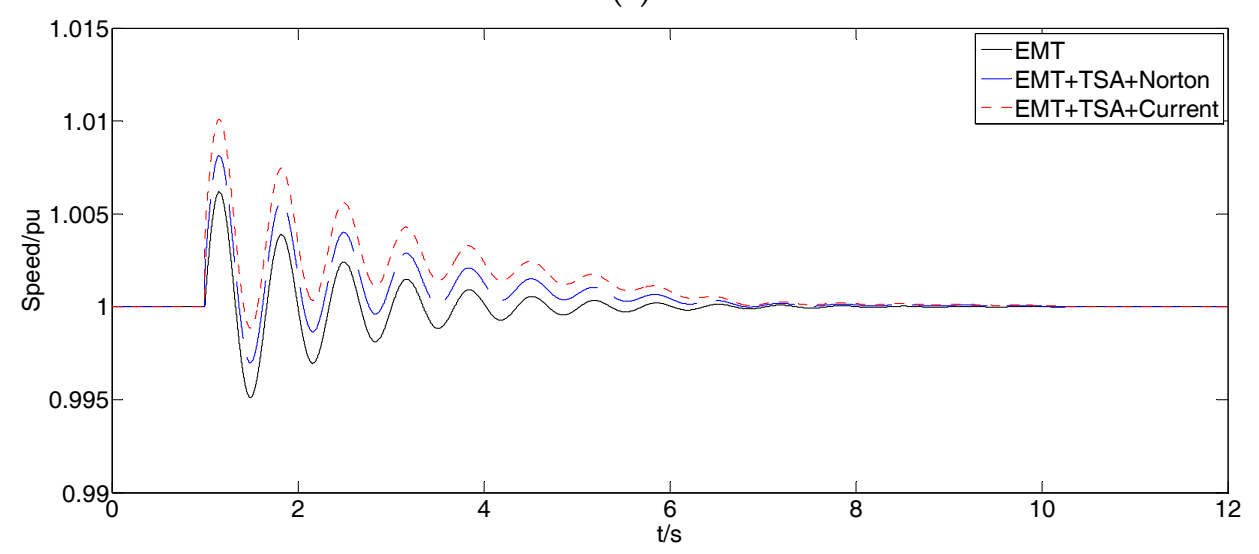

(b)

Figure 5. Verification of Norton equivalent accuracy. (a) Phase A voltage at bus 23; and, (b) Rotor speed of generator at bus 25 . 
It can be seen from Figure 5 that the hybrid simulation with the electromagnetic sub-network while using a Norton equivalent on the TSA side is closer to the EMT reference curve than the hybrid simulation using the current source equivalent. That is, the accuracy of the hybrid simulation is improved. It is proven that the method of obtaining the Norton equivalent of an electromagnetic sub-network on the TSA side is effective.

\subsubsection{Verification of Variable Area of Interest and Application}

To illustrate the effectiveness of the proposed hybrid real-time simulation method, two methods are designed for comparison: full EMT simulation, hybrid real-time simulation proposed in this paper. The hybrid real-time simulation method that is proposed in this paper is represented by the symbol $\mathrm{EMT}+\mathrm{TSA}+$ Variable. Only the test results of two cases are given.

\section{a. Case One}

Sub-network 7 and its affiliated area (sub-networks 2, 4, 8, 9, and 13) performs EMT simulation, and other parts perform TSA simulation. A $100 \mathrm{~ms}$ three-phase fault is applied to bus 37 . The phase A voltage of bus 37 and the rotor speed of the generator at bus 34 are as shown in Figure 6.

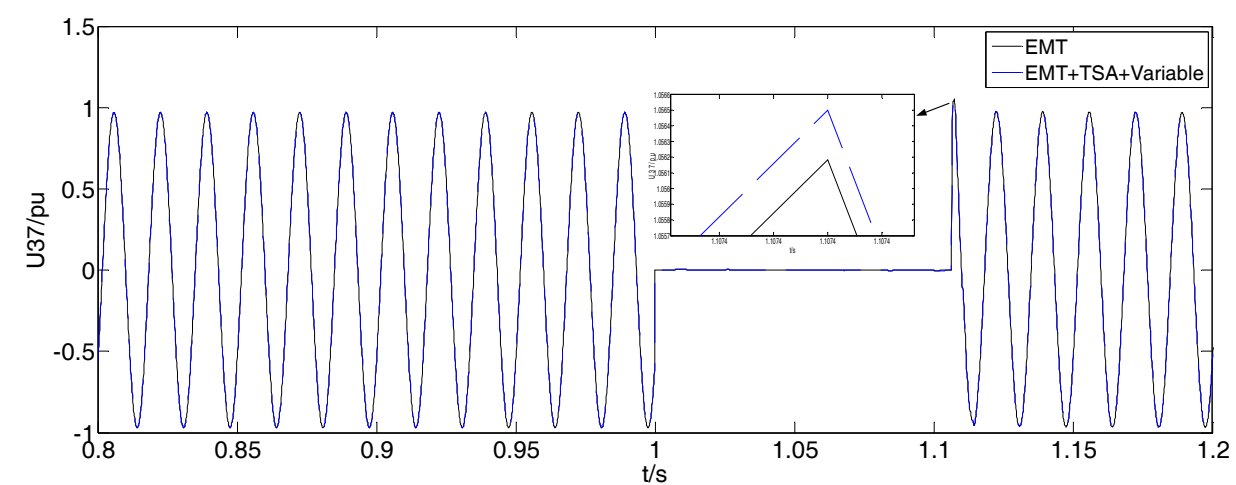

(a)

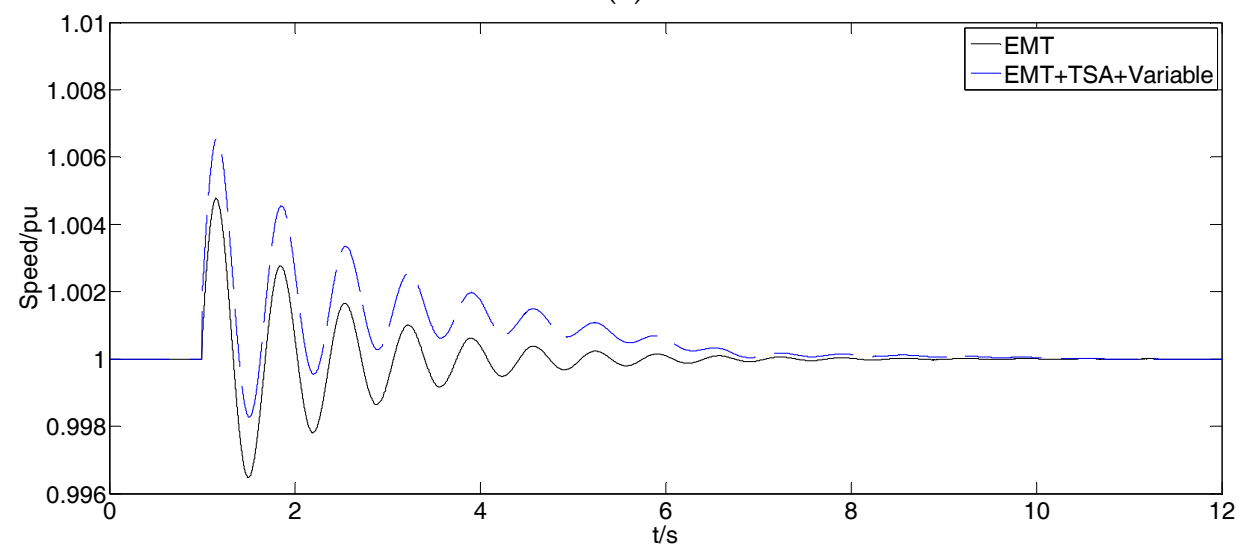

(b)

Figure 6. Comparison of simulation accuracy under case one. (a) Phase A voltage at bus 37 under case one; and, (b) Rotor speed of generator at bus 34 under case one.

\section{b. Case Two}

It can be seen from Figure 6 that the system basically reaches steady state at $t=12 \mathrm{~s}$, and the object that performs EMT simulation can be changed at this time. Occurring at $t=12 \mathrm{~s}$, sub-network 20 and its affiliated area (sub-networks 18, 19, 23, 24, 26, and 25) performs EMT simulation and other parts perform TSA simulation. A $100 \mathrm{~ms}$ three-phase fault is applied to bus 100. The phase A voltage of bus 100 and the rotor speed of the generator at bus 100 are as shown in Figure 7. 


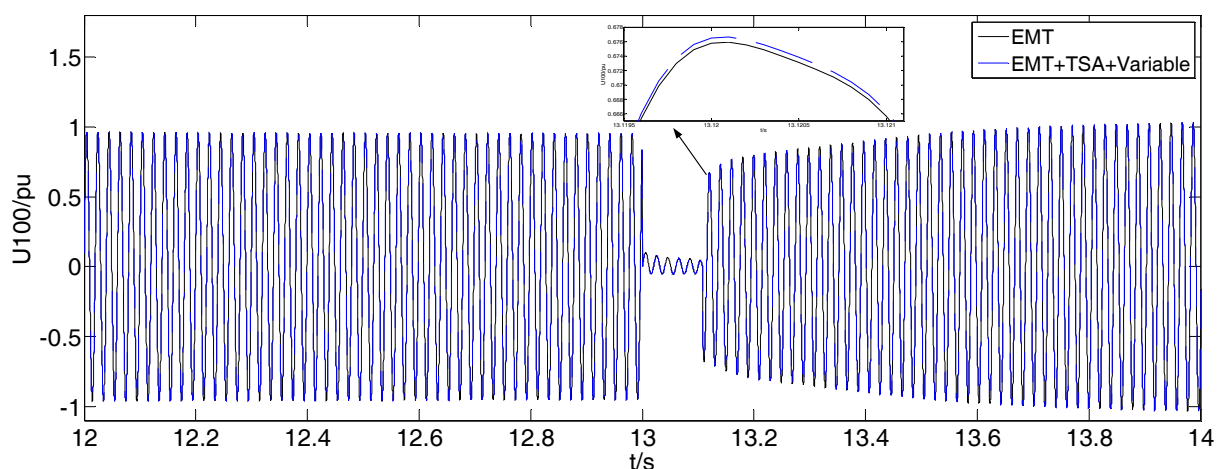

(a)

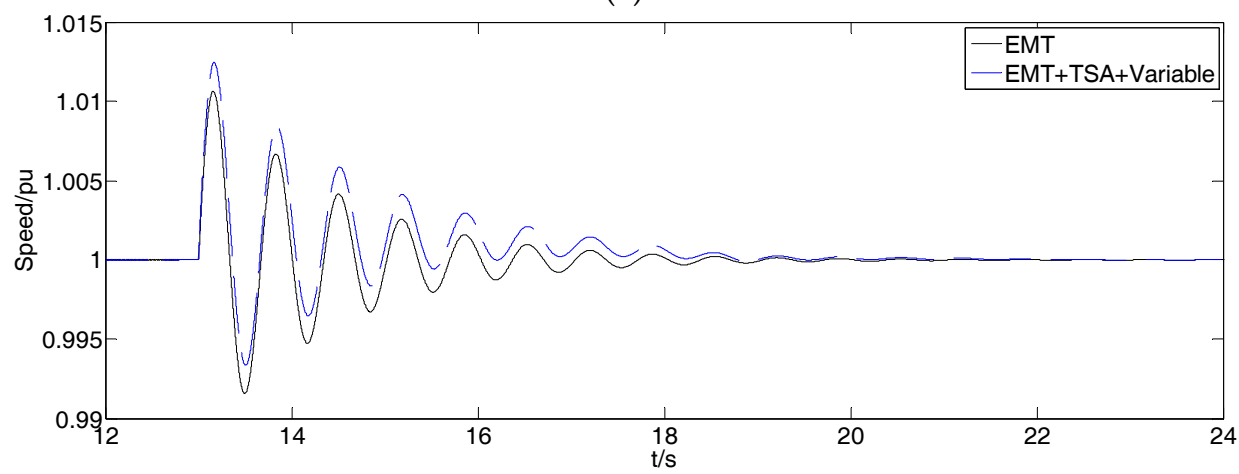

(b)

Figure 7. Comparison of simulation accuracy under case two. (a) Phase A voltage at bus 100 under case two; and, (b) Rotor speed of generator at bus 100 under case two.

Figures 6 and 7 show case one and case two are continuous in time. Figure 7 demonstrates that the waveform of the phase A voltage of bus 100 and the waveform of the rotor speed of the generator at bus 100 can maintain continuity when the area of EMT simulation is changed. This is because the system basically reaches steady state at $t=12 \mathrm{~s}$, and the EMT model and the TSA model of the same sub-network at steady state are equivalent. Through analyzing the simulation results, it can be seen that, although a serious three-phase short-circuit fault occurs at the boundary of the area of interest, the average difference [31] of the voltages at the fault points and the average difference of rotor speed are all within $0.05 \mathrm{pu}$. It is proven that the proposed method can ensure that the area of EMT simulation is flexibly changed and meets the requirements of simulation accuracy.

\section{Conclusions}

1. The general model of the contact system constructed by Norton equivalents can ensure the flexible change of the EMT simulation area, so that the EMT simulation of the entire system can be realized, and the continuity of the simulation variable waveform can be maintained, when the EMT simulation object is changed.

2. The proposed network partition scheme can be applied well to large-scale AC systems. Once the network is divided, the accuracy of the simulation can be guaranteed, even in the case of a serious three-phase short-circuit fault at any location in the area of interest. Thus, the method and criterion for determining the range of EMT simulation are effective. This is very important for realizing the hybrid real-time simulation of large-scale AC systems.

3. The modified Norton equivalent model of the electromagnetic sub-network that is used on the TSA side not only keeps the model of the contact system unchanged, but it also improves the simulation accuracy. 
Author Contributions: conceptualization, B.Z.; methodology, B.Z. and S.N.; software, S.N.; validation, S.N.; formal analysis, B.Z.; investigation, S.N. and Z.J.; resources, B.Z.; data curation, Z.J.; writing-original draft preparation, S.N.; writing-review and editing, B.Z. and S.N.; visualization, S.N. and Z.J.; supervision, B.Z.; project administration, B.Z.; funding acquisition, B.Z.

Funding: This research was funded by the National Natural Science Foundation of China, 51477114.

Conflicts of Interest: The authors declare no conflict of interest.

\section{References}

1. Faruque, M.O.; Strasser, T.; Lauss, G.; Jalili-Marandi, V.; Forsyth, P.; Dufour, C.; Dinavahi, V.; Monti, A.; Kotsampopoulos, P.; Martinez, J.A.; et al. Real-Time Simulation Technologies for Power Systems Design, Testing, and Analysis. IEEE Power Energy Technol. Syst. J. 2015, 2, 63-73. [CrossRef]

2. Guillaud, X.; Faruque, M.O.; Teninge, A.; Hariri, A.H.; Vanfretti, L.; Paolone, M.; Dinavahi, V.; Mitra, P.; Lauss, G.; Dufour, C.; et al. Applications of Real-Time Simulation Technologies in Power and Energy Systems. IEEE Power Energy Technol. Syst. J. 2015, 2, 103-115. [CrossRef]

3. Li, Y. Algorithm and Software Research on Real-Time Electromechanical Transient Simulation of Large-Scale Power System. Ph.D. Thesis, China Electric Power Research Institute, Beijing, China, 2003.

4. Le-Huy, P.; Sybille, G.; Giroux, P.; Loud, L.; Huang, J.; Kamwa, I. Real-time EMT and TS co-simulation based on hybrid line modelling. IET Gener. Trans. Distrib. 2017, 11, 2983-2990. [CrossRef]

5. Hariri, A.; Faruque, M.O. A Hybrid Simulation Tool for the Study of PV Integration Impacts on Distribution Networks. IEEE Trans. Sustain. Energy 2017, 8, 648-657. [CrossRef]

6. Wang, Z. Research of Interfaces Based on Real-Time Digital Simulation (RTDS) for Hybrid Real-Time Simulation of Electromagnetic-Electromechanical Transient Process. Master's Thesis, North China Electric Power University, Beijing, China, 2010.

7. Zhang, S.; Tong, L.; Xue, W.; Hong, C. Digital computer and RTDS based real time hybrid simulation. Autom. Electr. Power Syst. 2009, 33, 61-66.

8. Li, Q.; Zhang, J.; Xiao, X.; Li, W.; Wang, J. Electromechanical-EMT real-time simulation based on RTDS and its application to FACTS. Trans. China Electrotech. Soc. 2012, 27, 219-226.

9. Wang, D.; Tong, L.; Hong, C. Digital computer electromechanical transient and RTDS EMT hybrid real-time simulation system. Power Syst. Technol. 2008, 32, 42-46.

10. Reeve, J.; Adapa, R. A new approach to dynamic analysis of AC networks incorporating detailed modeling of DC systems. I. Principles and implementation. IEEE Trans. Power Deliv. 1988, 3, 2005-2011. [CrossRef]

11. Liu, W.; Hou, J.; Tang, Y.; Song, X.; Wang, L.; Fan, S. Electromechanical transient/EMT hybrid simulation method considering asymmetric faults. Proc. CSEE 2010, 30, 8-15.

12. Zhang, Y.; Wu, W.; Zhang, B.; Aniruddha, M.G. Frequency Dependent Network Equivalent Based Electromagnetic and Electromechanical Decoupled Hybrid Simulation. Proc. CSEE 2012, 32, 107-114.

13. Liu, Y.; Liang, X.; Min, Y.; Hu, M. An interface algorithm in power system electromechanical transient and EMT hybrid simulation. Autom. Electr. Power Syst. 2006, 30, 44-48.

14. Yang, Y.; Xiao, X.; Wang, H.; Liu, X.; Wu, L. Review and prospect of power system digital hybrid simulation technology. Electr. Power Autom. Equip. 2017, 37, 203-210.

15. Jia, X. Research and Implementation of Real-Time Digital Simulation Method of AC-DC Power System Based on RTDS. Ph.D. Thesis, North China Electric Power University, Hebei, China, 2009.

16. Wang, X.; Zhang, B.; Chen, M. Multi-rate Real-time Simulation Method Based on RTDS and FPGA Co-simulation Platform. Autom. Electr. Power Syst. 2016, 40, 144-150.

17. Zhu, X.; Zhou, X.; Tian, F.; Xu, D.; Huang, L.; Li, G. Hybrid electromechanical-electromagnetic simulation to transient process of large-scale power grid on the basis of ADPSS. Power Syst. Technol. 2011, 35, $26-31$.

18. Su, H.T.; Chan, K.W.; Snider, L.A.; Chung, T.S.; Fang, D.Z. Recent advancements in electromagnetic and electromechanical hybrid simulation. In Proceedings of the 2004 International Conference on Power System Technology, Singapore, 21-24 November 2004.

19. Reeve, J.; Adapa, R. A new approach to dynamic analysis of AC networks incorporating detailed modeling of DC systems. II. Application to interaction of DC and weak AC systems. IEEE Trans. Power Deliv. 1988, 3, 2012-2019. [CrossRef] 
20. Lin, X.; Gole, A.M.; Yu, M. A wide-band multi-port system equivalent for real-time digital power system simulators. IEEE Trans. Power Syst. 2009, 24, 237-249. [CrossRef]

21. Hu, Y.; Wu, W.; Zhang, B.; Guo, Q. Development of an RTDS-TSA hybrid transient simulation platform with frequency dependent network equivalents. In Proceedings of the 4th IEEE/PES Innovative Smart Grid Technologies Europe (ISGT EUROPE), Lyngby, Denmark, 6-9 October 2013.

22. Liang, Y.; Lin, X.; Gole, A.M.; Yu, M.; Zhang, Y.; Zhang, B. Comparisons of impact on the modeling detail on real time simulation of large power systems with HVDC. In Proceedings of the International Conference Power System Transients (IPTS), Delft, The Netherlands, 17-20 June 2011.

23. Zhang, S.; Liang, X.; Tong, L.; Hong, C. Key Technologies of the Power System Electromagnetic/Electromechanical Real-time Hybrid Simulation. Autom. Electr. Power Syst. 2008, 32, 89-96.

24. Zhang, Y.; Wu, W.; Zhang, B.; Aniruddha, M.G. Frequency dependent network equivalent for electromagnetic and electromechanical hybrid simulation. Proc. CSEE 2012, 32, 61-68.

25. Plumier, F.J.; Geuzaine, C.; Cutsem, T.V. On the convergence of relaxation schemes to couple phasor-mode and EMTs simulations. In Proceedings of the IEEE Power Energy Society General Meeting, National Harbor, MD, USA, 27-31 July 2014.

26. Plumier, F.J.; Geuzaine, C.; Cutsem, T.V. A multirate approach to combine EMTs and fundamental-frequency simulations. In Proceedings of the International Conference on Power System Transients (IPST), Vancouver, BC, Canada, 18-20 July 2013.

27. Aristidou, P.; Geuzaine, C.; Cutsem, T.V. Co-simulation of EMTs and phasor models: A relaxation approach. IEEE Trans. Power Del. 2016, 31, 2360-2369.

28. Oppenheim, A.V.; Schafer, R.W. Discrete-Time Signal Processing, 3rd ed.; Publishing House of Electronics Industry: Beijing, China, 2011.

29. Xia, D. Power System Analysis, 1st ed.; China Electrical Power Press: Beijing, China, 1995; pp. $49-51$.

30. Yang, Y.; Xiao, X.; Tao, S.; Chen, P. Multi-Port Interface Technique and Application of Electromagnetic and Electromechanical Transient Hybrid Real-Time Simulation. Trans. China Electrotech. Soc. 2017, 32, 231-240.

31. Plumier, F.J.; Aristidou, P.; Geuzaine, C.; Cutsem, T.V. A relaxation scheme to combine phasor-mode and EMTs simulations. In Proceedings of the Power Systems Computation Conference (PSCC), Wroclaw, Poland, 18-22 August 2014.

(C) 2018 by the authors. Licensee MDPI, Basel, Switzerland. This article is an open access article distributed under the terms and conditions of the Creative Commons Attribution (CC BY) license (http:/ / creativecommons.org/licenses/by/4.0/). 